\title{
THE RF SYSTEM OF THE LNLS INJECTOR SYNCHROTRON
}

\author{
C. Pardine, P. F. Tavares and R. H. A. Farias, LNLS, Campinas, Brazil
}

\section{Abstract}

We present the RF system for the $500-\mathrm{MeV}$ booster of the LNLS synchrotron light source. The heart of the RF system is a home-made narrow-band MOSFET based solid state amplifier which delivers up to $900 \mathrm{~W}$ RF power to the $476 \mathrm{MHz}$ Elettra-type RF cavity of the booster. The amplifier is made up of 5 conveniently combined modules, each module being able to deliver up to $230 \mathrm{~W}$ at $476 \mathrm{MHz}$. The system also includes the usual feedback loops for amplitude and phase controls.

\section{INTRODUCTION}

The new injector for the Brazilian Synchrotron Light Source (LNLS) consists of a 120-MeV LINAC and a 500-MeV booster synchrotron [1]. The booster synchrotron was recently commissioned and the whole facility will be open for users on July 2001 [2]. Besides the primary task as the new injector for the $1.37 \mathrm{GeV}$ storage ring the machine is also intended to be used as a small storage ring for beam physics studies. The main characteristic of this 34-m circumference booster are the long ramping cycles, i.e., the low repetition rate it operates at, thus avoiding the need for special vacuum chambers. At present, more than $40 \mathrm{~mA}$ can be injected into the booster and about $15 \mathrm{~mA}$ can be ramped to 500 $\mathrm{MeV}$ at $0.17 \mathrm{~Hz}$ repetition rate.

Given the relatively low RF power requirements of the booster (less than $1 \mathrm{~kW}$ ), solid state power amplifiers have been developed as a cost-effective alternative to a

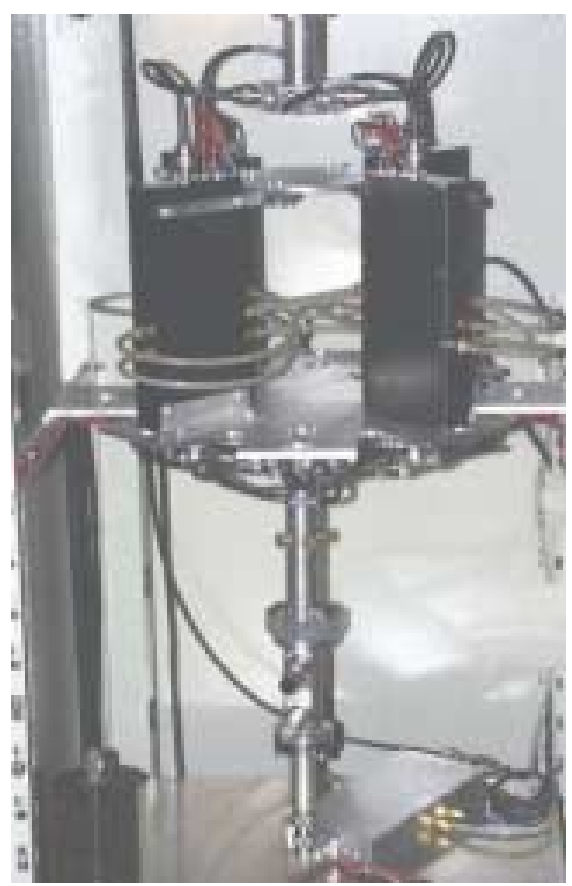

Figure 1: $900 \mathrm{~W}$ RF power amplifier assembly. commercial klystron or tetrode tube. This development has benefited greatly from the expert advice of RF engineers at the Synchrotron Radiation Facility LURE, in France [2]. By the end of 1999, a prototype amplifier module reached a record of $250 \mathrm{~W} \mathrm{CW}$ power with $60 \%$ efficiency@476 MHz. In its final configuration 9 modules will be used to produce up to $1.6 \mathrm{~kW}$. In the current stage we have been operating a $900 \mathrm{~W}$ 5-module assembly.

\section{THE RF SYSTEM}

The $900 \mathrm{~W}$ solid state RF power supply now operating at the LNLS Booster is formed by 5 amplifier modules, each one delivering up to $230 \mathrm{~W}$ of $\mathrm{RF}$ power at $476 \mathrm{MHz}$. These modules are the basic units of the amplifier and, by a convenient combination of these modules high power may be achieved. The topology used for the modules and for summing up the power is based on the one now under development at LURE $[3,4]$. The 5 -module assembly is shown in Figure 1. One of the modules is used as pre-amplifier to the other four modules. A Fluke RF generator provides the reference signal that drives the whole RF system of the booster and of the storage ring. A commercial $53 \mathrm{~dB}$-gain $550 \mathrm{~F}$ Kalmus amplifier amplifies a sample of that signal and can deliver up to $25 \mathrm{~W}$ to the pre-amplifier module. A power divider upstream the pre-amp is used to feed each of the four modules of the assembly. Finally, the output power of these modules is combined and delivered through a $7 / 8$ " Heliax coaxial cable to the RF cavity. In the $900 \mathrm{~W}$ configuration special care has to be taken in order to prevent the pre-amp from delivering too much power to the modules upstream. In the final stage of the amplifier the same pre-amp will be used to drive 8 modules.

\subsection{Solid-state Amplifiers}

The core of the amplifier is a gold metallised RF Power MOSFET that can deliver up to $250 \mathrm{~W}$ at $476 \mathrm{MHz}$. The transistor used is a D1029UK, with push-pull configuration, made by Semelab plc. Class AB operation is used in order to obtain a compromise between efficiency and linearity. Each module includes a built-in isolator at the output port terminated with a $65 \mathrm{~W} R F$ terminator. The isolator was introduced for reliability and stability purposes since without it the amplifier is not unconditionally stable. The modules have been operating at lower power levels, delivering up to $230 \mathrm{~W}$ under normal operation. Each module has about $9 \mathrm{~dB}$ gain and the average efficiency is $55 \%$ at maximum power. The gain is within $\pm 2 \mathrm{~dB}$ range from that value for $30 \mathrm{~dB}$ span 

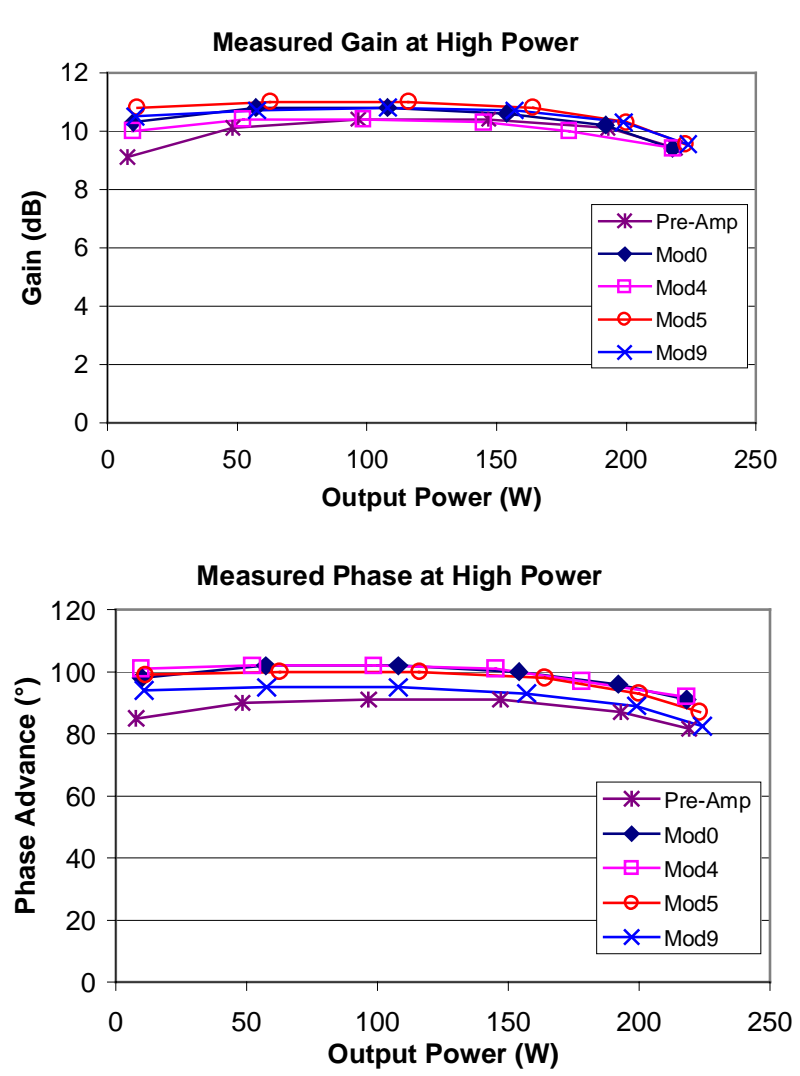

Figure 2: Gain and Phase Advance measurements for the modules used in the $900 \mathrm{~W}$ assembly.

of the input power and that encompasses the full operation range of the modules.

A full characterisation of each module was carried out including gain, phase advance and stability measurements. Stability measurements were performed at

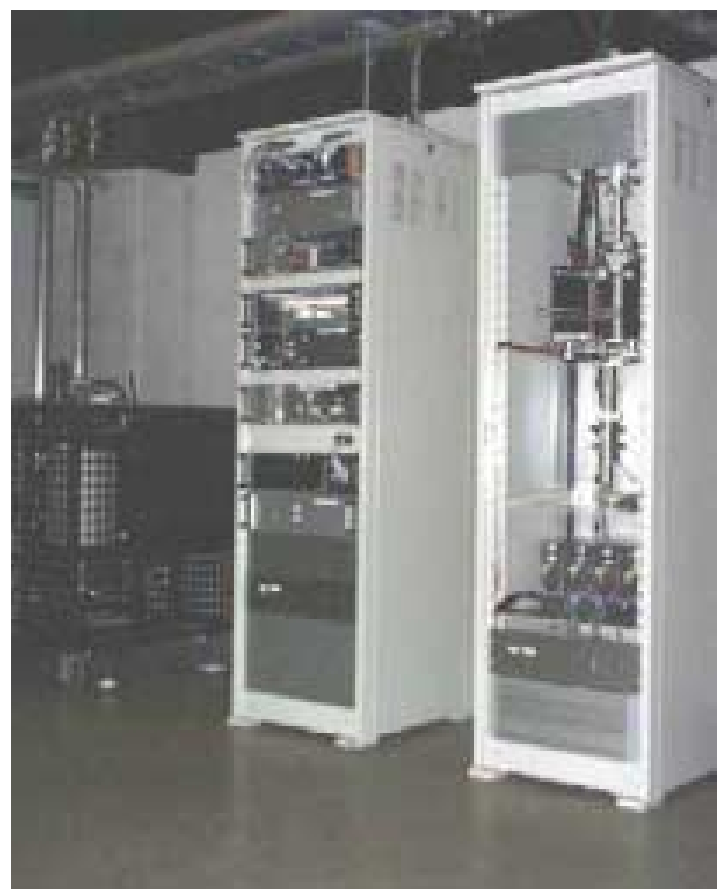

Figure 3: (Left) RF control and amplifier racks; (Right) Amplifier module low power only and each module was adjusted in order to show unconditional stability and gain protection in excess of $5 \mathrm{~dB}$. The elimination of the dangerous self-excited modes was achieved by installing comb filters in parallel to the source impedance and by slightly changing the reactance of the matching transmission lines that take the input power to the power transistor. The phase advance of each module was adjusted in order to fall within a maximum difference of $10^{\circ}$ between modules thus limiting the gain degradation due to phase difference to less than $0.05 \mathrm{~dB}$ (Fig. 2). Phase and gain measurements were performed at high power using an Agilent 8753ES Network Analyser set to A/B mode and with its internal sweep generator turned off.

\subsection{Other RF Components}

Home made power combiners and dividers are used to feed the 4-module set of amplifiers and to sum up the output power. These are narrow-band devices with very low insertion loss (below $0.1 \mathrm{~dB}$ ). The divider has a very good impedance matching at the input port with 1.03 VSWR.

The bi-directional 38-dB couplers used for monitoring the output and reflected power to the modules were also home made. The insertion loss of the couplers is smaller than $0.05 \mathrm{~dB}$ and the isolation between the two ports larger than $35 \mathrm{~dB}$.

\subsection{RF Cavity}

The Booster RF cavity is a bell-shaped Elettra-type cavity that operated in the main storage ring for two years. The power is inductively coupled to the cavity by a high power loop feedthrough. The cavity has openings for vacuum pumping and for diagnostics. Three antennas are

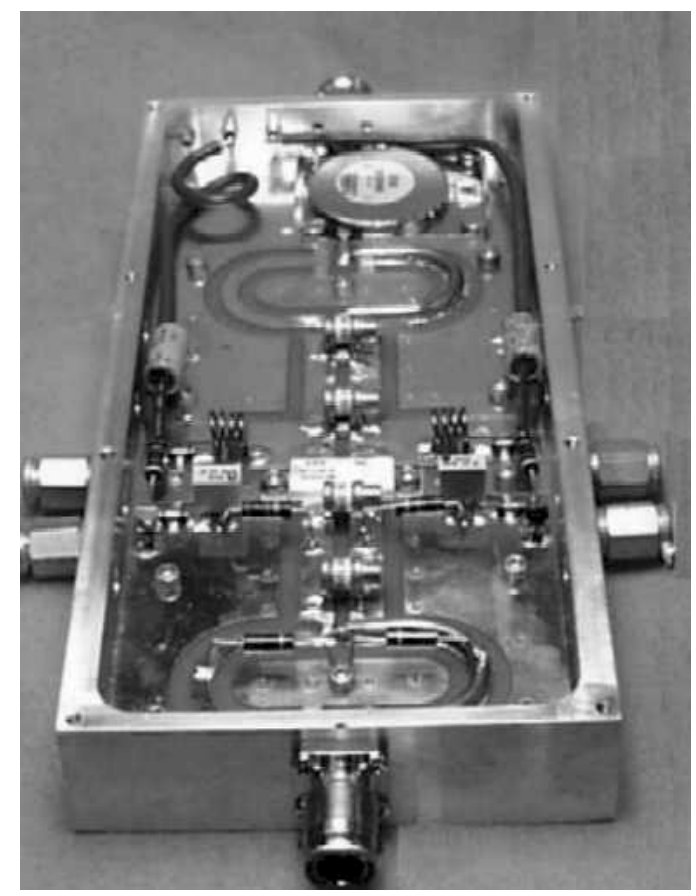

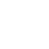




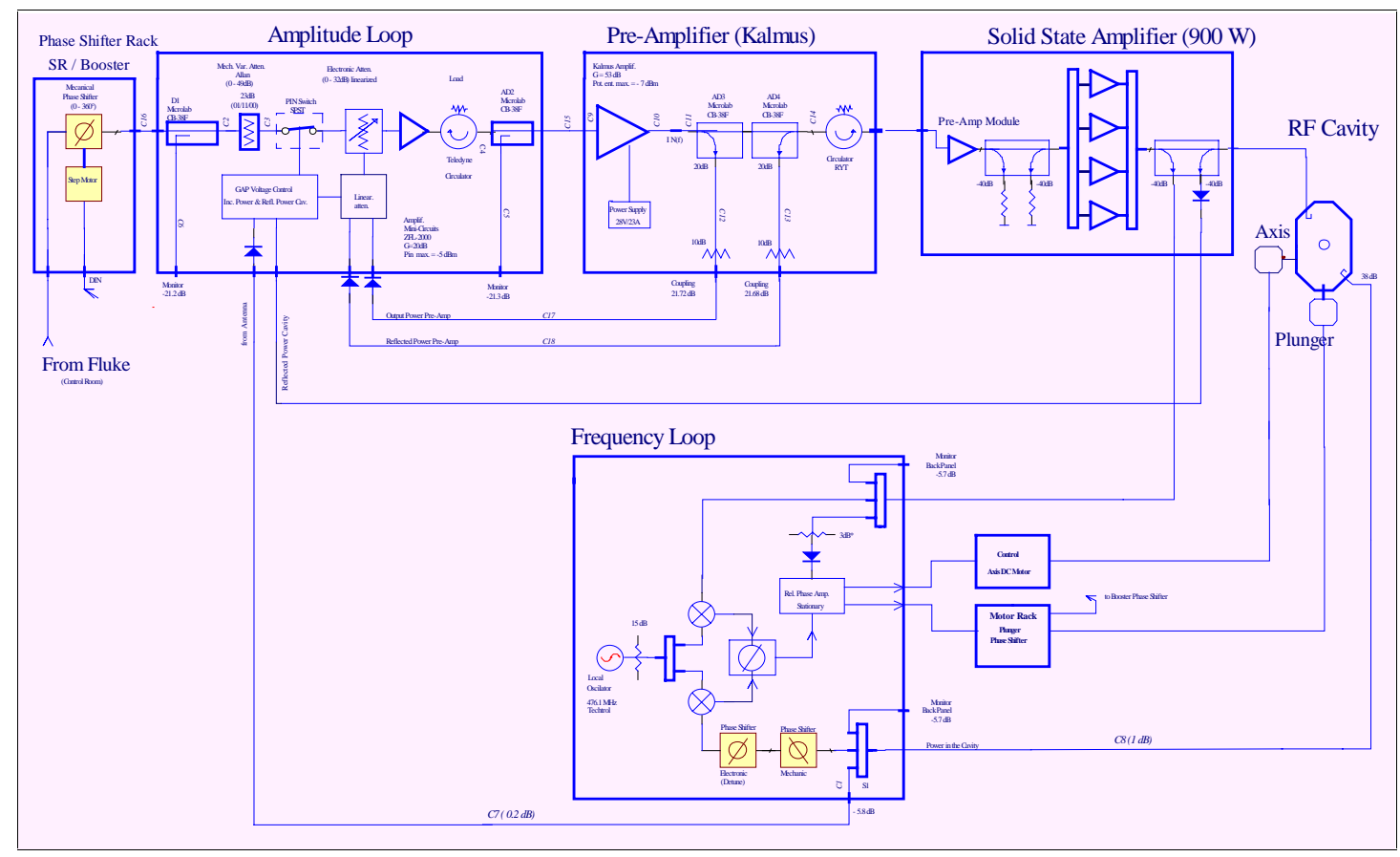

Figure 4: Schematic diagram of the RF system.

installed for feedback and monitoring purposes. The cavity has two mechanisms for tuning control that work via geometrical deformation of the cavity walls. Besides the conventional motor-driven plunger there is also a mechanical system that squeezes and stretches the cavity axially. The frequency loop controls these two movements in order to bring the frequency of the fundamental resonant mode to the requested set point. A wide-ranging study of the resonant modes of the cavity was carried out. All the HOM up to $2.1 \mathrm{GHz}$ were identified. Measurements were performed in order to map the variation of the resonant frequencies with temperature, and plunger and axis positions.

\subsection{RF Control System}

The RF system as a whole comprises subsystems that are responsible for the control of the amplifier, the feedback loops, the interlock system and for the temperature control of the RF cavity. There are two feedback loops operating on the cavity control. The frequency loop mentioned above controls the tune of the cavity by changing the plunger position and by deforming the cavity. The RF signals from the cavity and from the amplifier are downmixed to the $100-\mathrm{kHz}$ range and the phase detection is performed with a HCT4046 PLL. The amplitude loop controls the accelerating voltage in the cavity gap by changing the input power to the amplifier. A fast feedback loop for beam loading compensation is to be installed in the near future. The temperature control system can keep the temperature of the cavity to within $\pm 0.1^{\circ} \mathrm{C}$ around the operation temperature. The whole system is computer controlled and is fully integrated into the SR-Booster control program.

\section{CONCLUSION}

The RF system of the booster has been operating since last November with different versions of the amplifier, with 1, 2 and now 5 modules. The system has shown itself very reliable but special care had to be taken in order to protect it against large reflected power from the cavity. The large beam loading effect and the slow response of the tuning system result in large reflected power from the cavity in the interval between ejection and the next injection into the booster. In order to minimise these problems water cooling of the circulator container and the increase of the terminators power tolerance to $400 \mathrm{~W}$ have been introduced in the modules. The main characteristics of the solid state amplifier are: it is always ready to operate, it is very simple to maintain, and its modular structure, that allows expansion by simply adding new module cells.

\section{ACKNOWLEDGEMENTS}

We are deeply indebted to eng. Ti Ruan (LURE) for his invaluable suggestions and support.

\section{REFERENCES}

[1] A.R.D. Rodrigues et al, "Design of a Booster for the Brazilian Synchrotron Light Source (LNLS)," EPAC'98,Stockholm, June 1998.

[2] P.F. Tavares et al, "Commissioning of the LNLS $500 \mathrm{MeV}$ Booster Synchrtron", at this Conference.

[3] T. Ruan et al, " $500 \mathrm{MHz} 1 \mathrm{~kW}$ CW MOSFET Amplifier". Super-Aco/98-03.

[4] J.-M. Godefroy et al, "MOSFET RF Power Amplifier for Accelerator Applications", EPAC'98, Stockholm, June 1998 